\title{
An Upgraded Cryogenic Test Stand for HL-LHC Cryo-Assemblies
}

\section{R. Rabehl, O. al Atassi, G. Chlachidze, S. Feher, S. Koshelev, and S. Ranpariya - Fermilab}

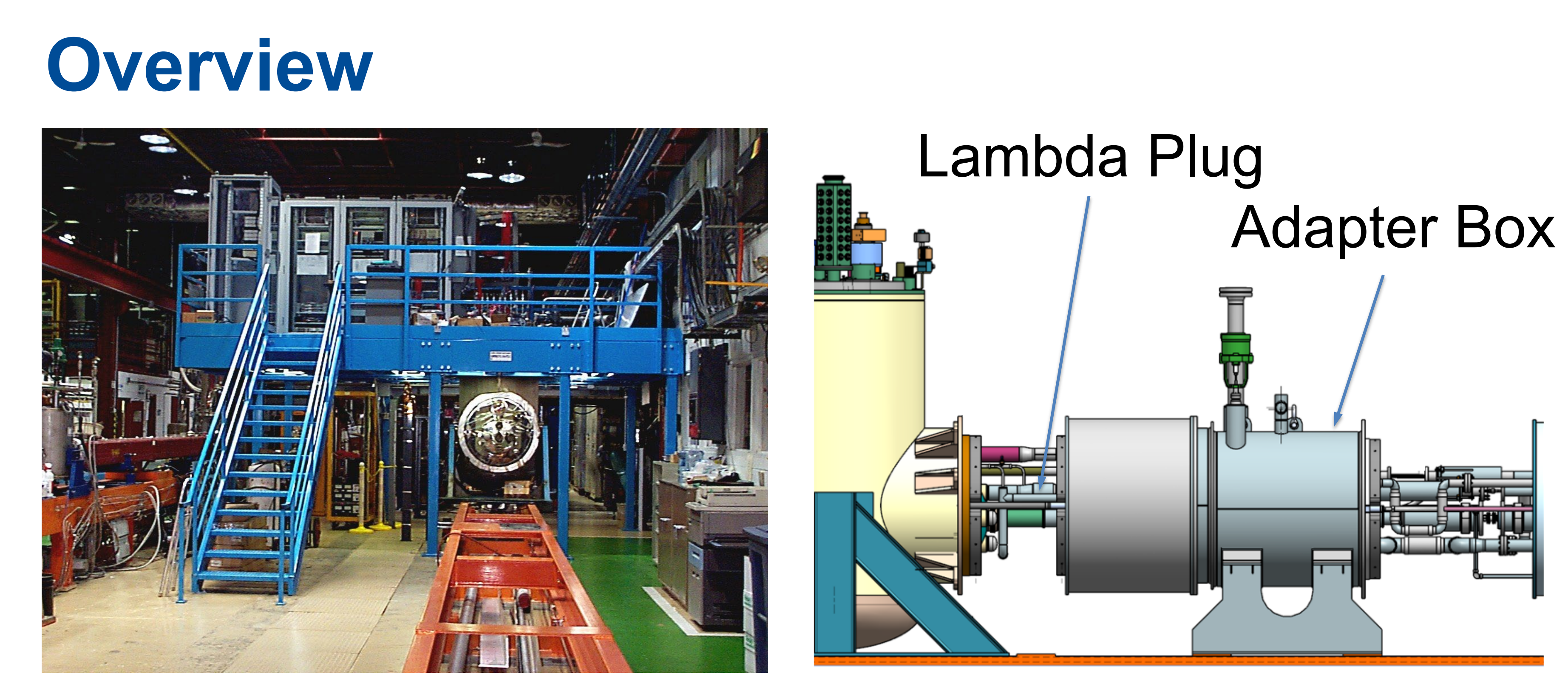

The original test stand, shown at the above left, included a cryogenic feed box that directly interfaced with LHC interaction region quadrupoles. An upgrade of the test stand was required to accommodate the testing requirements of HL-LHC cryo-assemblies. As shown in the above right, this upgrade included an Adapter Box, a lambda plug, and redesigned interconnects.

\section{Adapter Box and Lambda Plug}
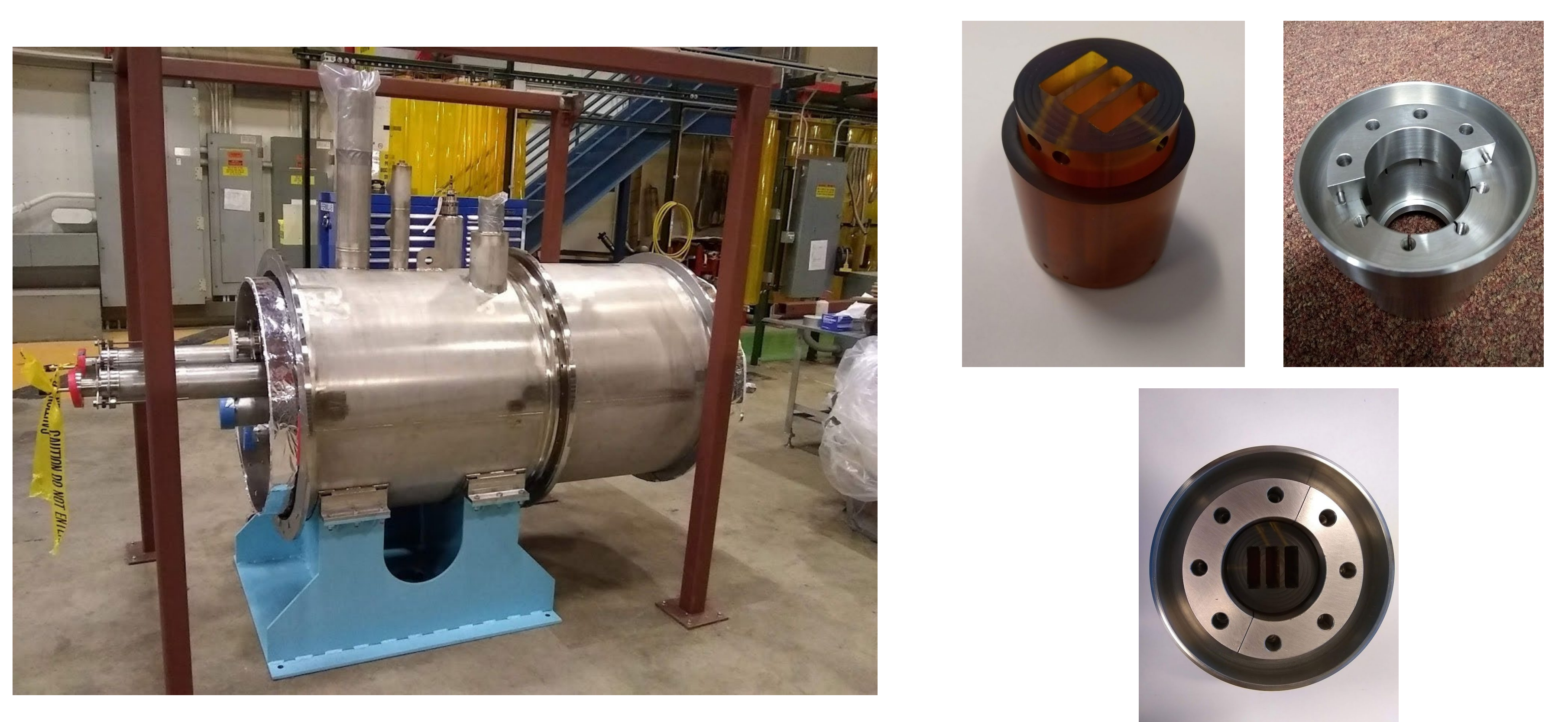

The Adapter Box provides an interface between the feed box and the new HL-LHC cryoassemblies. An LHC lambda plug design was modified to incorporate additional buswork. Together, they provide the interface between $4.5 \mathrm{~K}$ and $1.9 \mathrm{~K}$ helium volumes.

\section{Piping}

The test stand upgrade included piping system upgrades, including:

- New U-tubes supplying cryogens (left),

- An LN2 bath and mass flow controllers for controlled cool-down/warm-up (center), and

- New cool-down return, quench recovery, and pressure safety valve systems (right).

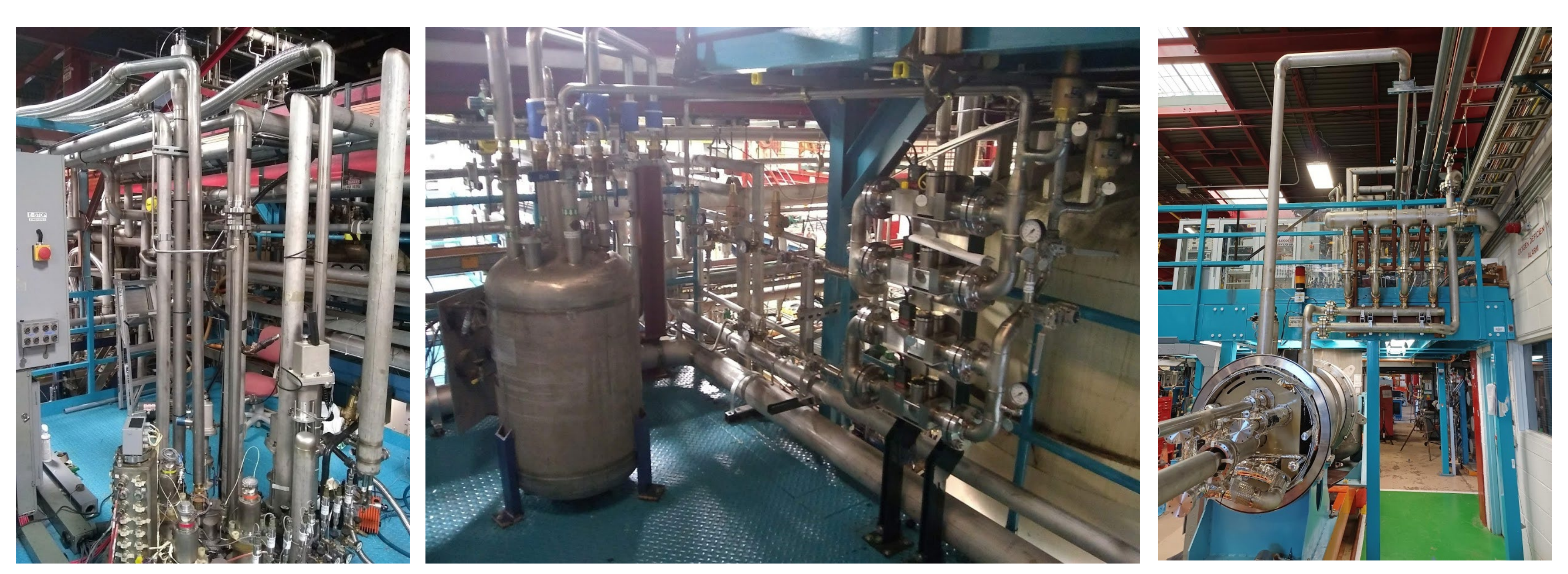

\section{Zero Magnet Test}

A zero magnet test was successfully conducted to verify functionality of systems before the start of production testing. The upgraded test stand was cooled down and all combinations of the three vapor-cooled current leads in the feed box were powered.

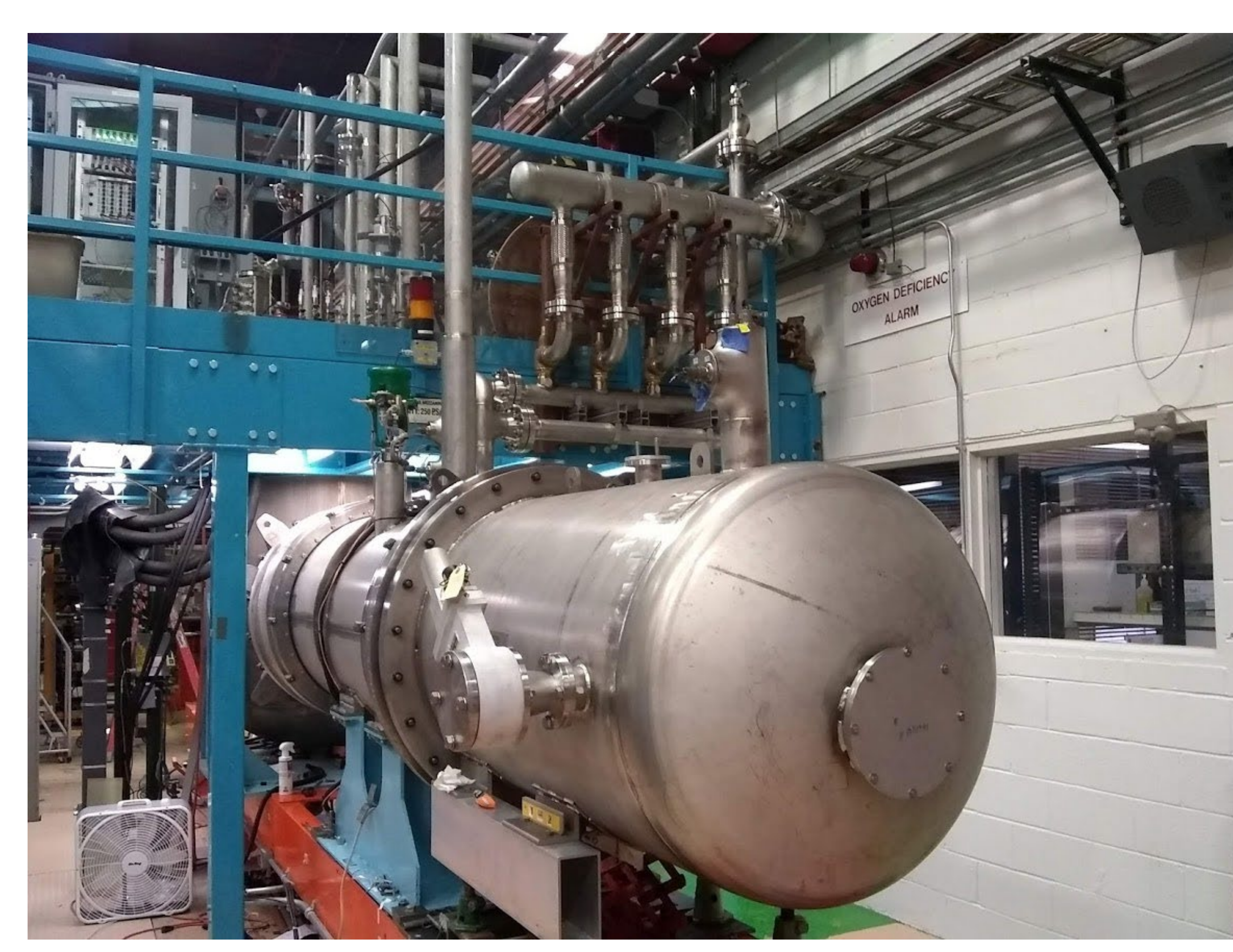

Process Controls System

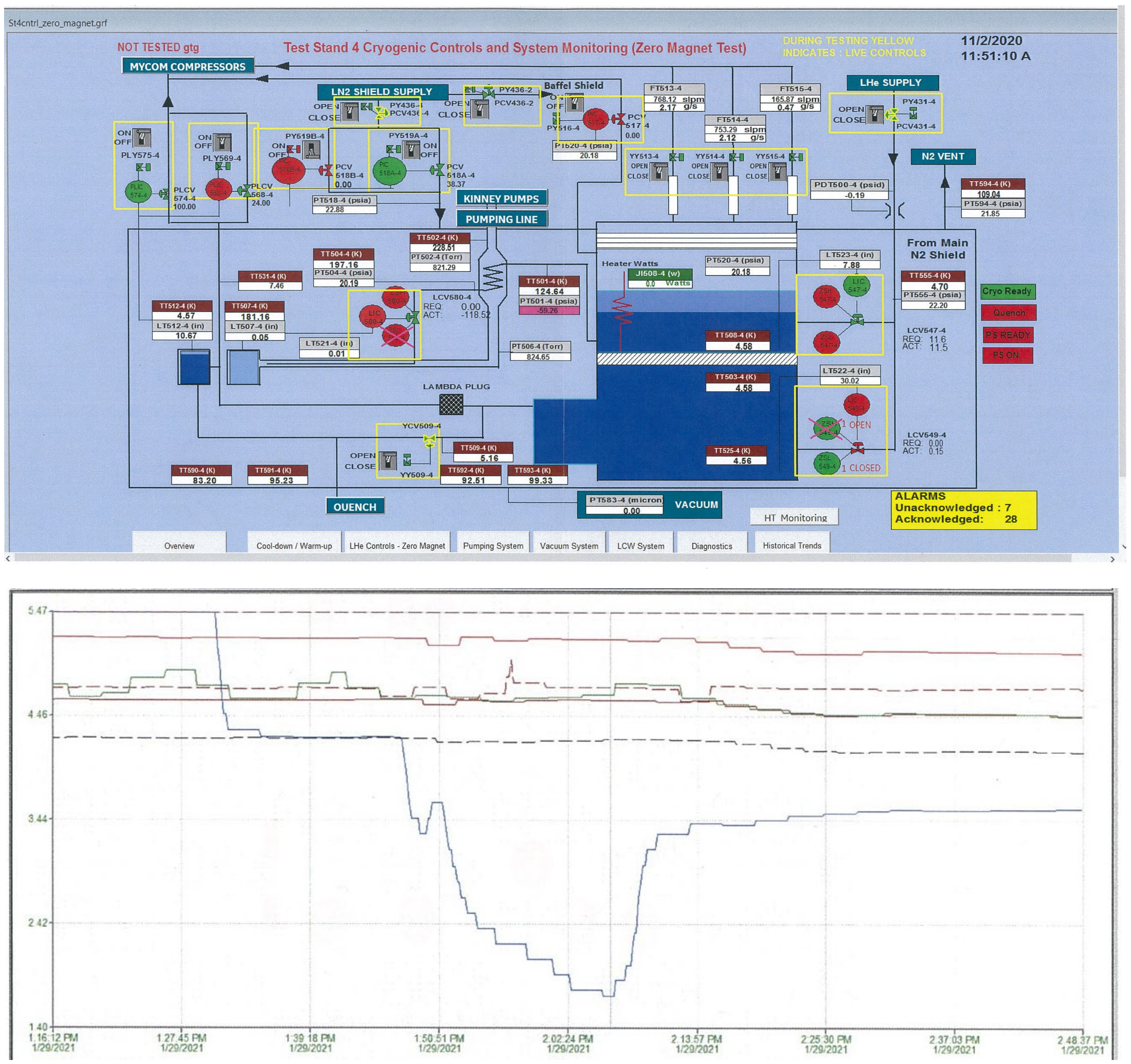

A Siemens 545 PLC was replaced with a Siemens S7 PLC for cryogenic process control. The original operator interface screens and historical data collection using Intellution Fix32 were upgraded to GE Digital iFix. A test stand monitoring screen and a historical plot showing cooldown of the upgraded pumping line to $1.9 \mathrm{~K}$ (blue line) are shown above.

\section{Conclusion}

The Fermilab horizontal test stand has been upgraded and successfully operated in a zero magnet test configuration in preparation for production testing of HL-LHC cryo-assemblies.

This manuscript has been authored by Fermi Research Alliance, LLC under Contract No. DE-AC02-07CH11359 with the U.S. Department of Energy, Office of Science, Office of High Energy Physics. 